\title{
A Comparison between Windowing FIR Filters for Extracting the EEG Components
}

\author{
Mawia A Hassan ${ }^{1 *}$, Elwathiq A Mahmoud ${ }^{2}$, Abdalla H Abdalla² and Ahmed M Wedaa ${ }^{2}$ \\ ${ }^{1}$ Biomedical Engineering Department, Sudan University of Science and Technology, Khartoum, Sudan \\ ${ }^{2}$ Medical Engineering Department, University of Science and Technology, Omdurman, Sudan
}

\begin{abstract}
Electroencephalogram (EEG) is a test used to detect abnormalities related to electrical activity of the brain. In this work different finite impulse response filter (FIR) windows methods were used to extract EEG signal to its basic components (Delta wave, Theta wave, Alpha wave and Beta wave). The comparison between these windowing methods were done by computing the Fourier transform, power spectrum, SNR, the main-lobe, and the side-lobe. The results show the best main-lobe is for rectangular window, the best side-lobe is for Kaiser $\beta(12)$ and the best SNR is for Hanning. Also the best window according to main-lobe, side-lobe and SNR is Kaiser $\beta$ (12).
\end{abstract}

Keywords: Electroencephalogram (EEG); Finite impulse response filter (FIR); SNR; Main-lobe; Side-lobe; Electrodes

\section{Introduction}

Electroencephalogram (EEG) used to measure abnormalities in the brain, which related to electrical activity (Figure 1). This measurement tracks and records brain wave patterns by using electrodes are placed on the scalp, and then signals go to a computer to record the results. Abnormal patterns in EEG indicate seizures and other problems [1-6]. The important reason for using EEG is to diagnose and show seizure disorders. Also EEGs help to help to identify causes of other problems like sleep disorders and changes in behaviour. To evaluate the brain activity after head injury or before heart or liver transplantation the EEG is a good choice [3]. The electrodes in the conventional scalp EEG are placed on the scalp with a conductive gel or paste which is led to reduce impedance due to dead skin cells. When used on highdensity arrays of electrodes are needed many systems use caps or nets which electrodes are embedded in it. The Electrodes locations and names depend on an international 10-20 system for most clinical and research applications (except when high-density arrays are used). About 19 recording electrodes (plus ground and system reference) are used in most clinical applications. If the spatial resolution is the aim of interest further electrodes can be added for a particular area of the brain and this in clinical or research application. Up to 256 electrodes can be used in High-density arrays, it may become more-or-less evenly spaced around the scalp. Differential amplifier can be used to connect the electrode (one amplifier per pair of electrodes). The other input of each differential amplifier is connected by a common reference electrode. The job of these amplifiers is to amplify the voltage between the reference and active electrode and (1,000-100,000 times, or 60-100 $\mathrm{dB}$ voltage gain). Most EEGs systems nowadays are digital, the signal is amplified and pass through ADC and anti-aliasing filter. The sampling rate between $256-512 \mathrm{~Hz}$ in the clinical scalp EEG; in some research applications, it may use sampling rates of up to $20 \mathrm{kHz}$. For adult EEG signal is about $10 \mu \mathrm{V}$ to $100 \mu \mathrm{V}$ when the measuring from the scalp and is about $10-20 \mathrm{mV}$ when measuring from subdural electrodes $[3,4]$. The fundamental components of the EEG system are shown in Figure 1.

The basic signals in the EEG are: Delta wave with a frequency range up to $4 \mathrm{~Hz}$. This wave is the highest in amplitude and the slowest waves [4] $\mathrm{m}$ theta wave has the frequency range from $4 \mathrm{~Hz}$ to $7 \mathrm{~Hz}$. Theta is seen normally in young children [4-8], Alpha wave is the frequency range from $7 \mathrm{~Hz}$ to $14 \mathrm{~Hz}$. Beta wave is the frequency range from $15 \mathrm{~Hz}$ to about $30 \mathrm{~Hz}$. In areas of cortical damage this wave may be reduced or absent. It is the dominant rhythm in patients who are alert or anxious or who have their eyes open [9-11].

One of the most basic elements in a digital signal processing system is the finite impulse response (FIR) filter. It can guarantee a linear phase frequency characteristic with frequency characteristic. Also the unit impulse response is finite. In this work different finite impulse response filter (FIR) windows methods were used to extract the EEG signal to its basic components (Delta wave, Theta wave, Alpha wave and Beta wave) [12-15].

\section{Methods}

In this work comparison between different FIR windowing filters to extract EEG signal to its basic components (delta, theta, alpha and beta). The steps are:

1) EEG signals (Delta, theta, alpha and beta) were extracted by band-pass filter using Rectangular, Hamming, Hanning, Kaiser and Blackman windows (Table 1).

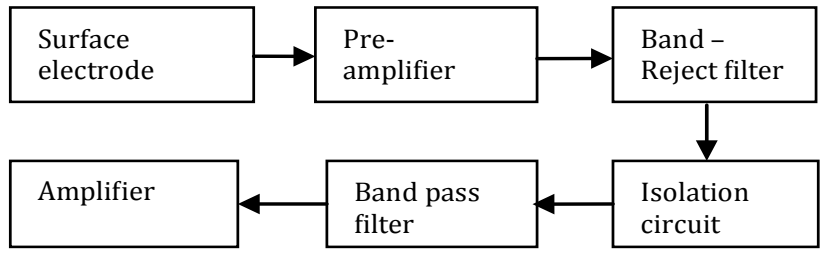

Figure 1: EEG Block Diagram

*Corresponding author: Mawia A Hassan, Biomedical Engineering Department Sudan University of Science and Technology, Khartoum, Sudan, Tel: +249912273789; E-mail: mawiaahmed@sustech.edu

Received October 09, 2015; Accepted November 19, 2015; Published November 29, 2015

Citation: Mahmoud EA, Abdalla AH, Wedaa AM, Hassan MA (2015) A Comparison between Windowing FIR Filters for Extracting the EEG Components. J Biosens Bioelectron 6: 191. doi:10.4172/2155-6210.1000191

Copyright: (c) 2015 Mahmoud EA, et al. This is an open-access article distributed under the terms of the Creative Commons Attribution License, which permits unrestricted use, distribution, and reproduction in any medium, provided the original author and source are credited. 
Citation: Mahmoud EA, Abdalla AH, Wedaa AM, Hassan MA (2015) A Comparison between Windowing FIR Filters for Extracting the EEG Components. J Biosens Bioelectron 6: 191. doi:10.4172/2155-6210.1000191

\begin{tabular}{|c|c|}
\hline Window & Equation \\
\hline Rectangular & $\mathrm{Y}[\mathrm{n}]=\sum_{\mathrm{k}=\mathrm{m} 1}^{\mathrm{m} 2} \mathrm{bkx}[\mathrm{n}-\mathrm{k}]$ \\
\hline Hamming & $\mathrm{R}[\mathrm{n}]=\left\{\begin{array}{lr}1 & 0 \ll n \ll l-1 \\
0 & \text { otherwise }\end{array}\right.$ \\
\hline Kaiser & $W[n]=\left\{\begin{array}{l}\frac{i 0 \sqrt{1-\left(\frac{n-m}{m}\right)^{2}}}{I(\beta)} 0 \ll n \ll 1-1 \\
0 \quad \text { otherwise }\end{array}\right.$ \\
\hline Hanning & $\mathrm{W}[\mathrm{n}]=\left\{\begin{array}{c}0.5-0.5 \cos \frac{2 \pi \mathrm{n}}{1-1} o \ll n \ll l-1 \\
0 \quad \text { otherwise }\end{array}\right.$ \\
\hline Blackman & $\mathrm{W}[\mathrm{n}]=\left\{\begin{array}{cc}0.42-0.5 \cos \frac{4 \pi \mathrm{n}}{1-1} 0.08 \cos \frac{4 \pi \mathrm{n}}{1-1} 0 \ll \mathrm{n} \ll 1-1 \\
0 \quad \text { otherwise }\end{array}\right.$ \\
\hline
\end{tabular}

Table 1: Windowing functions.

\begin{tabular}{|c|c|c|c|}
\hline Window & Main-lobe -3 db & Side-lobe db & Snr db \\
\hline Rectangular & 0.0059 & -13.3 & -55.49 \\
\hline Hamming & 0.0044 & -42.7 & -55.03 \\
\hline Hanning & 0.0049 & -31.5 & -139.74 \\
\hline Kaiser beta 5 & 0.0044 & -36.8 & -55.73 \\
\hline Kaiser beta 8 & 0.0037 & -58.6 & -63.90 \\
\hline Kaiser beta 12 & 0.0033 & -89.9 & -66.69 \\
\hline Blackman & 0.0037 & -58.1 & -67.47 \\
\hline
\end{tabular}

Table 2: Main-lobes, side-lobes and SNR ratio for delta signal.

2) The length of each window was calculated using equation 1 (Table 2).

Where $\Delta \mathrm{F}_{\mathrm{w}}$ is the frequency resolution, $\mathrm{G}$ is the window factor, $\mathrm{F}_{\mathrm{s}}$ is the sampling frequency and $\mathrm{L}$ is the window length.

3) Compute the Fourier transform of each wave after extraction.

4) Compute the original power spectrum.

5) Compute the SNR for all signals.

\section{Results and Discussions}

Table 2 is shown the length of each FIR window using equation 1.

Figures 2-5 show the comparisons between the extracted the basic EEG components using rectangular, Hamming, Hanning, Kaiser (5, 8 and 12) and Blackman.

Table 3 show the comparison between main-lobes, side-lobes and SNR for delta signal filtered by windowing method.

Table 4 show the comparison between main-lobes, side-lobes and SNR for theta signal filtered by windowing method.

Table 5 show the comparison between main-lobes, side-lobes and SNR for alpha signal filtered by windowing method

Table 6 show the comparison between main-lobes, side-lobes and SNR for beta signal filtered by windowing method.

As can be shown best main-lobe is for rectangular window, the best side-lobe is for Kaiser $\beta$ (12) and the best SNR is for Hanning. Also

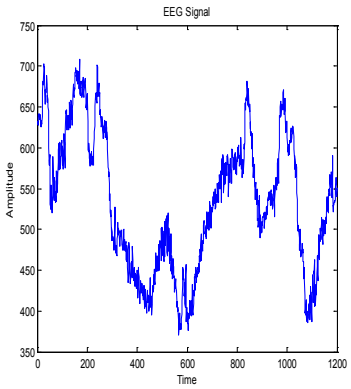

(a)

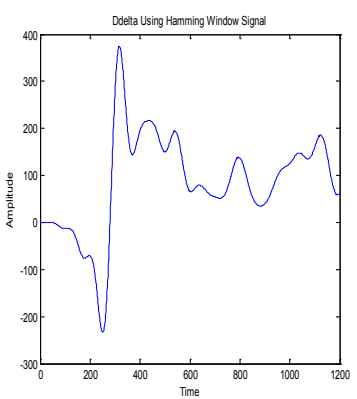

(c)

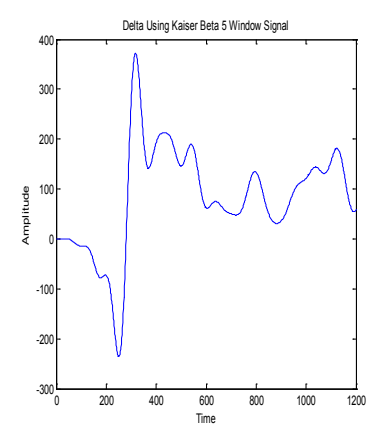

(e)

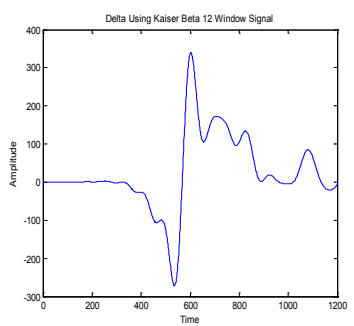

(g)

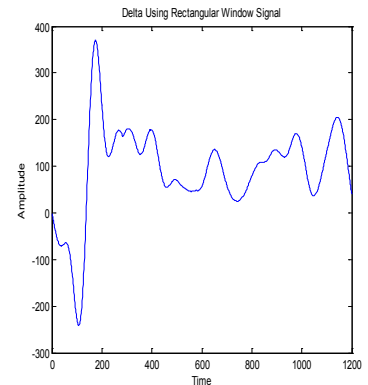

(b)

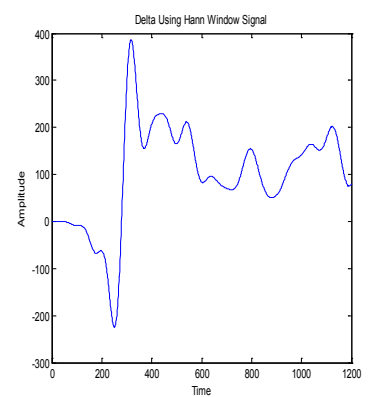

(d)

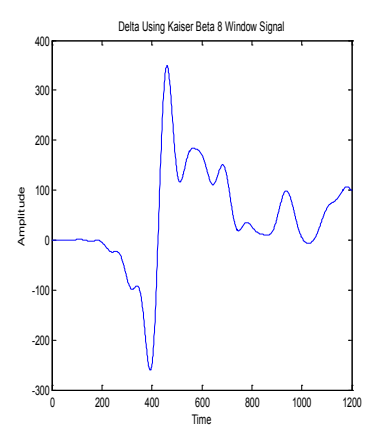

(f)

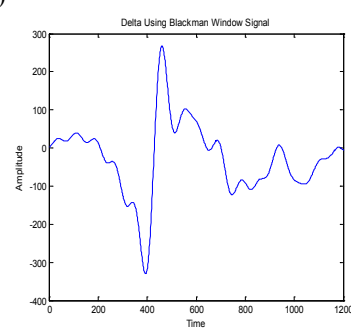

(h)
Figure 2: Comparison Between Extracted Delta Wave, (A) Eeg Signal, (B) Using Rectangular, (C) Using Hamming, (D) Using Hanning , (E) Using Kaiser $B=5$, (F) Using Kaiser $B=8,(G)$ Using Kaiser $B=12,(H)$ Using Blackman. 


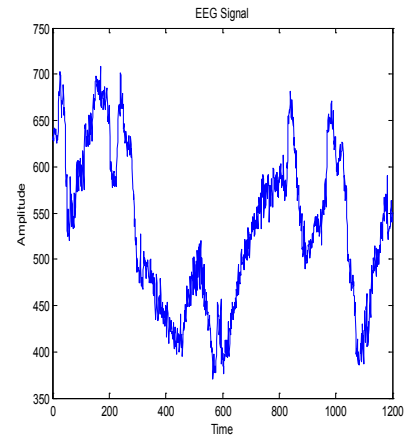

(a)

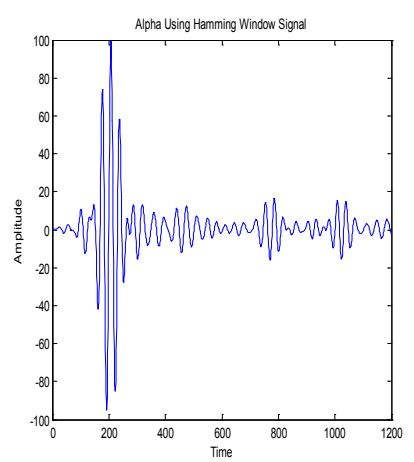

(c)

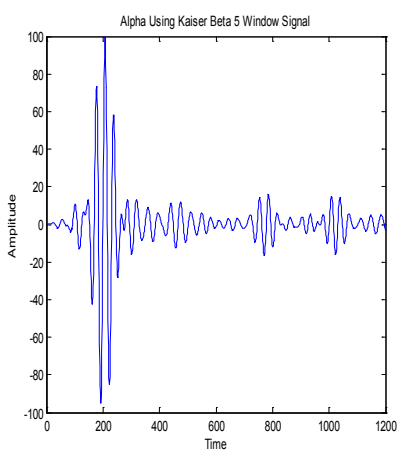

(e)

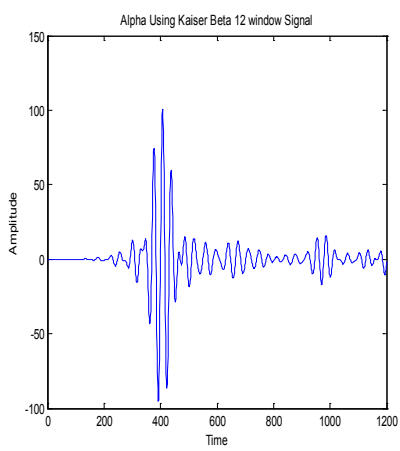

(g)

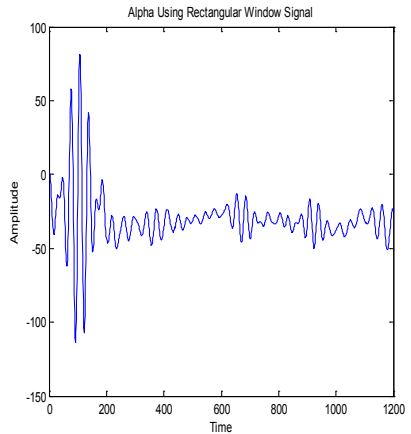

(b)

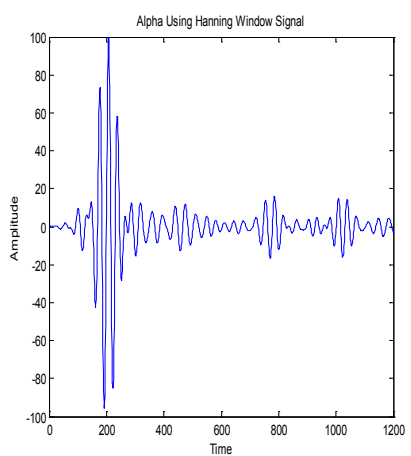

(d)

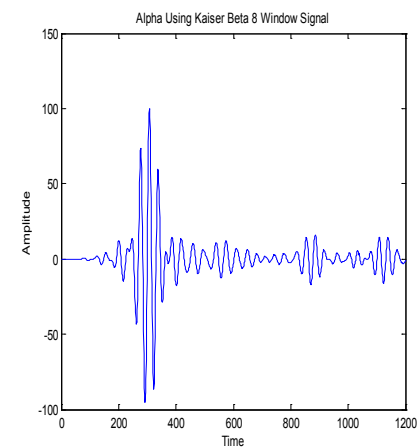

(f)

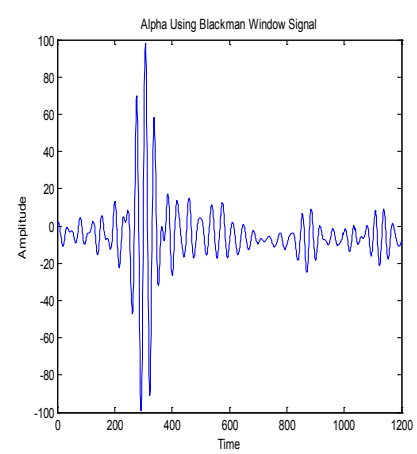

(h)

Figure 3: Comparison Between Extracted Theta Wave, (A) Eeg Signal, (B) Using Rectangular, (C) Using Hamming, (D) Using Hanning, (E) Using Kaiser B=5, (F) Using Kaiser $B=8,(G)$ Using Kaiser $B=12,(H)$ Using Blackman. 


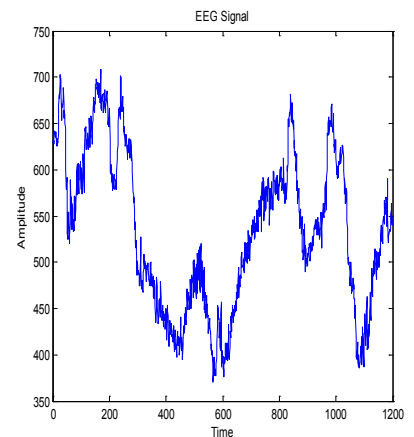

(a)

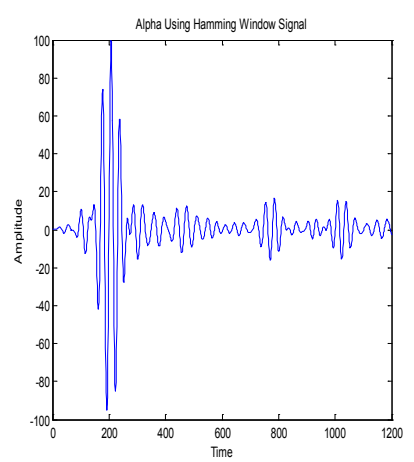

(c)

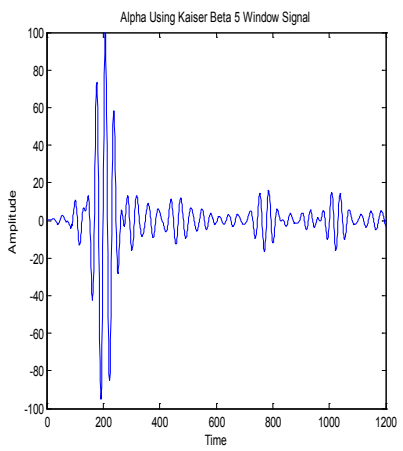

(e)

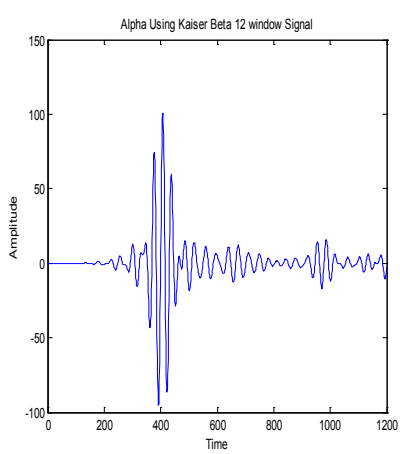

(g)

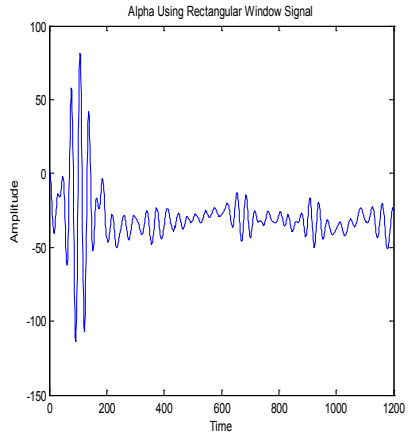

(b)

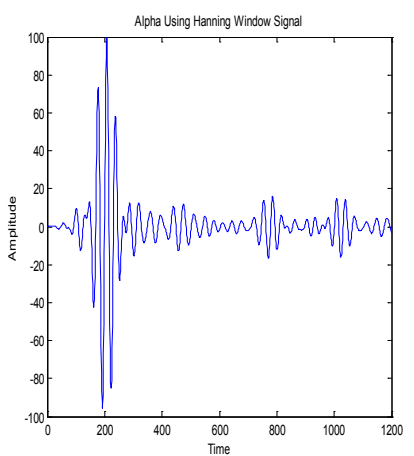

(d)

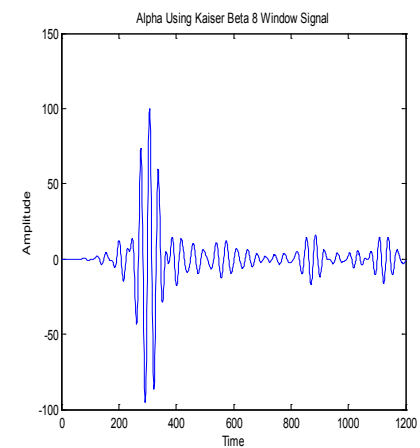

(f)

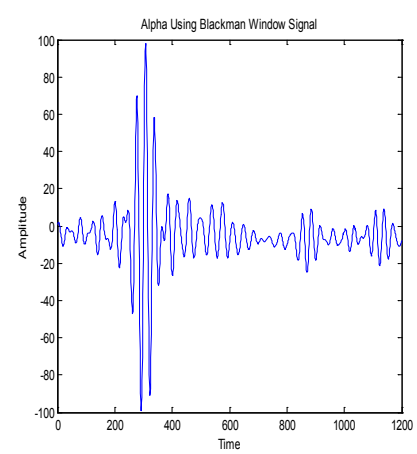

(h)

Figure 4: Comparison Between Extracted Alpha Wave, (A) Eeg Signal, (B) Using Rectangular, (C) Using Hamming, (D) Using Hanning, (E) Using Kaiser B=5, (F) Using Kaiser $B=8,(G)$ Using Kaiser $B=12,(H)$ Using Blackman. 


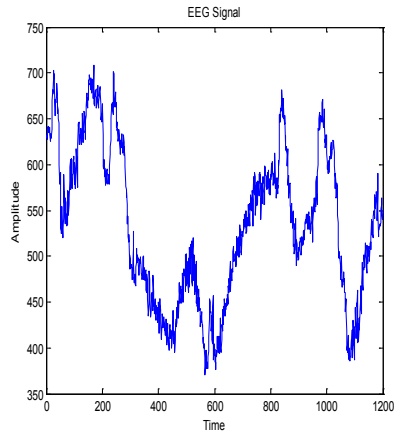

(a)

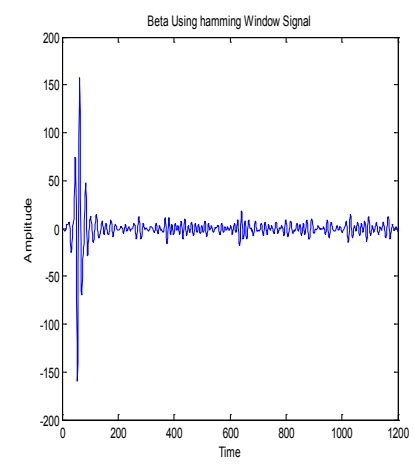

(c)

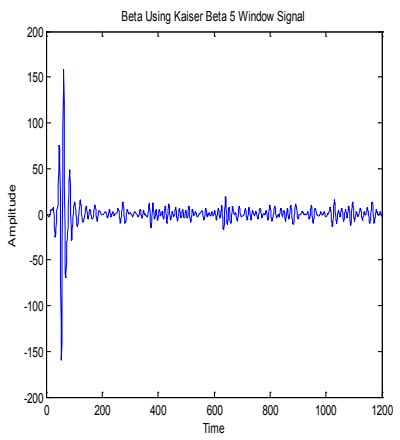

(e)

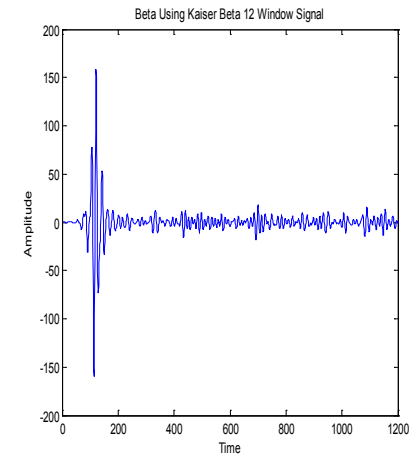

(g)

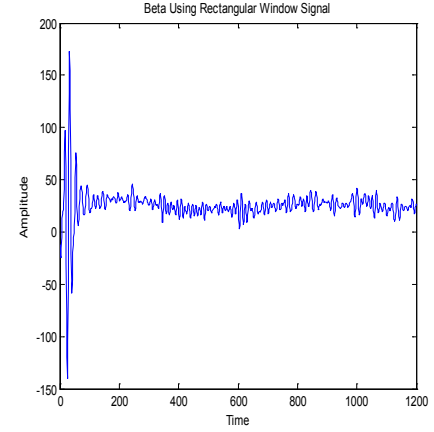

(b)

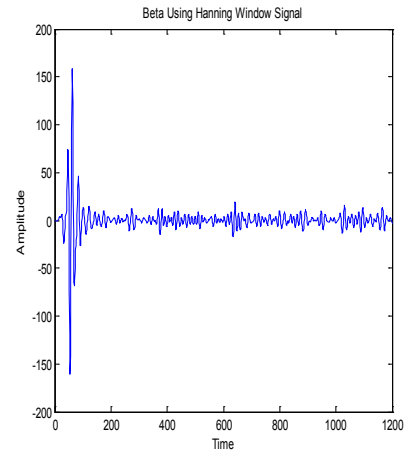

(d)

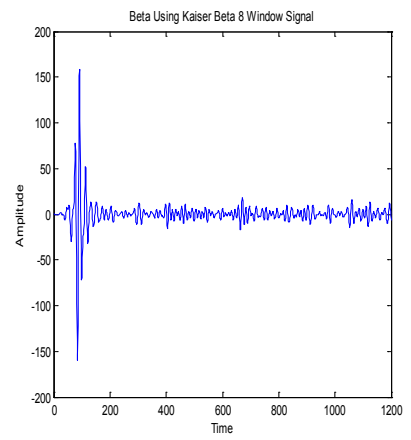

(f)

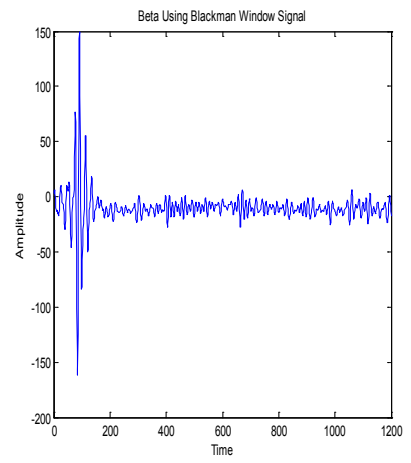

(h)

Figure 5: Comparison Between Extracted Beta Wave, (A) Eeg Signal, (B) Using Rectangular, (C) Using Hamming, (D) Using Hanning , (E) Using Kaiser B=5, (F) Using Kaiser $B=8,(G)$ Using Kaiser $B=12$, $(H)$ Using Blackman. 
Citation: Mahmoud EA, Abdalla AH, Wedaa AM, Hassan MA (2015) A Comparison between Windowing FIR Filters for Extracting the EEG Components. J Biosens Bioelectron 6: 191. doi:10.4172/2155-6210.1000191

Page 6 of 6

\begin{tabular}{|c|c|c|c|c|c|}
\hline \multirow{2}{*}{\multicolumn{2}{|c|}{ Windows }} & \multicolumn{4}{|c|}{ Signals } \\
\hline & & Delta & Theta & Alpha & Beta \\
\hline \multicolumn{2}{|c|}{ Rectangular } & 57 & 49 & 39 & 11 \\
\hline \multicolumn{2}{|c|}{ Hamming } & 115 & 99 & 79 & 23 \\
\hline \multicolumn{2}{|c|}{ Hanning } & 115 & 99 & 79 & 23 \\
\hline \multirow{3}{*}{ Kaiser $\beta$} & \multirow{3}{*}{$\begin{array}{c}5 \\
8 \\
12\end{array}$} & 115 & 100 & 79 & 23 \\
\hline & & 171 & 149 & 119 & 35 \\
\hline & & 227 & 199 & 159 & 47 \\
\hline \multicolumn{2}{|c|}{ Blackman } & 171 & 149 & 119 & 35 \\
\hline
\end{tabular}

Table 3: The length of each FIR window.

\begin{tabular}{|c|c|c|c|}
\hline Window & Main-lobe-3 db & Side-lobe db & SNR db \\
\hline Rectangular & 0.0068 & -13.3 & -97.51 \\
\hline Hamming & 0.0048 & -42.7 & -122.64 \\
\hline Hanning & 0.0054 & -31.5 & -122.59 \\
\hline Kaiser beta 5 & 0.0049 & -36.8 & -122.59 \\
\hline Kaiser beta 8 & 0.0042 & -58.6 & -122.94 \\
\hline Kaiser beta 12 & 0.0037 & -83.9 & -123.09 \\
\hline Blackman & 0.0042 & -58.1 & -121.76 \\
\hline
\end{tabular}

Table 4: Main-lobes, side-lobes and SNR ratio for theta signal.

\begin{tabular}{|c|c|c|c|}
\hline Window & Main-lobe-3 db & Side-lobe db & SNR db \\
\hline Rectangular & 0.0088 & -13.3 & -55.49 \\
\hline Hamming & 0.0063 & -42.7 & -139.74 \\
\hline Hanning & 0.0068 & -31.5 & -139.74 \\
\hline Kaiser beta 5 & 0.0063 & -36.8 & -139.70 \\
\hline Kaiser beta 8 & 0.0051 & -58.6 & -139.23 \\
\hline Kaiser beta 12 & 0.0046 & -90.0 & -139.41 \\
\hline Blackman & 0.0034 & -58.1 & -67.47 \\
\hline
\end{tabular}

Table 5: Main-lobes, side-lobes and SNR for alpha signal.

\begin{tabular}{|c|c|c|c|}
\hline Window & Main-lobe -3 db & Side-lobe db & SNR db \\
\hline Rectangular & 0.0273 & -13.3 & -115.80 \\
\hline Hamming & 0.0215 & -42.6 & -142.01 \\
\hline Hanning & 0.0239 & -31.5 & -141.96 \\
\hline Kaiser beta 5 & 0.0219 & -37.0 & -141.93 \\
\hline Kaiser beta 8 & 0.0176 & -58.5 & -141.49 \\
\hline Kaiser beta 12 & 0.0156 & -90.2 & -141.39 \\
\hline Blackman & 0.0186 & -58.1 & -133.32 \\
\hline
\end{tabular}

Table 6: Main-lobes, side-lobes and SNR ratio for beta signal.

the best window according to main-lobe, side-lobe and SNR is Kaiser $\beta(12)$.

\section{Conclusion}

In this study a comparisons between rectangular, Hamming, Hanning, Kaiser (5, 8 and12) and Blackman to extracted the EEG signal were shown. The results shown the best main-lobe is for rectangular window, the best side-lobe is for Kaiser $\beta$ (12) and the best SNR is for Hanning. Also the best window according to main-lobe, side-lobe and SNR is Kaiser $\beta$ (12).

\section{References}

1. Scarabino T, Salvolini U (2006) Atlas of Morphology and Functional Anatomy of the Brain, Naples-Italy.

2. Peng S (2013) Design and analysis of FIR filters based on Matlab. Electrical Engineering, Linnaeus University.

3. http //kidshealth.org/

4. John JW (2010) Medical Instrumentation Application and Design, 4th Edition, Wiley.

5. Steven WS (2003) The scientist and enginreer's, guide to digital signal processing, California technical publishing.

6. Niedermeyer E, Ernst Niedermeyer, Silva FL (2004) Electroencephalography: Basic Principles, Clinical Applications and Related Fields, Lippincot Williams \& Wilkins.

7. Towle, Vernon L, Bolaños J, Suarez D, Levin DN (1993) "The spatial location of EEG electrodes Locating the best-fitting sphere relative to cortical anatomy", Electroencephalography and Clinical Neurophysiology 86: 1-6.

8. Cahn BR, Polich J (2006) Meditation states and traits: EEG, ERP and neuroimaging studies. Psychol Bull 132: 180-211.

9. Niedermeyer E (1997) Alpha rhythms as physiological and abnormal phenomena. Int J Psychophysiol 26: 31-49.

10. Feshchenko VA, Reinsel RA, Veselis RA (2001) Multiplicity of the alpha rhythm in normal humans. J Clin Neurophysiol 18: 331-344.

11. Pfurtscheller G, Lopes da Silva FH (1999) Event-related EEG/MEG synchronization and desynchronization: Basic principles. Clin Neurophysiol 110: $1842-1857$

12. Aurlien H, Gjerde IO, Aarseth JH, Eldøen G, Karlsen B, et al. (2004) EEG background activity described by a large computerized database. Clin Neurophysiol 115: 665-673.

13. Maan MS (2007) "EEG Waves Classifier using Wavelet Transform and Fourier Transform”. International Journal of Biomedical Sciences 1: 85-90.

14. Al-Fahoum AS, Al-Fraihat AA (2014) Methods of EEG signal features extraction using linear analysis in frequency and time-frequency domains. ISRN Neurosci 2014: 730218.

15. Mohan Kumar CE, Dharani Kumar SV (2014) "Wavelet Based Feature Extraction Scheme of Electroencephalogram". International Journal of Innovative Research in Science, Engineering and Technology 3: 908-913. 